\title{
Nonlinear analysis of a cross-coupled quadrature harmonic oscillator
}

\author{
Djurhuus, Torsten; Krozer, Viktor; Vidkjær, Jens; Johansen, Tom Keinicke
}

Published in:

I E E E Transactions on Circuits and Systems Part 1: Regular Papers

Link to article, DOI:

10.1109/TCSI.2005.853586

Publication date:

2005

Document Version

Publisher's PDF, also known as Version of record

Link back to DTU Orbit

Citation (APA):

Djurhuus, T., Krozer, V., Vidkjær, J., \& Johansen, T. K. (2005). Nonlinear analysis of a cross-coupled quadrature harmonic oscillator. I E E E Transactions on Circuits and Systems Part 1: Regular Papers, 52(11), $2276-2285$. https://doi.org/10.1109/TCSI.2005.853586

\section{General rights}

Copyright and moral rights for the publications made accessible in the public portal are retained by the authors and/or other copyright owners and it is a condition of accessing publications that users recognise and abide by the legal requirements associated with these rights.

- Users may download and print one copy of any publication from the public portal for the purpose of private study or research.

- You may not further distribute the material or use it for any profit-making activity or commercial gain

- You may freely distribute the URL identifying the publication in the public portal 


\title{
Nonlinear Analysis of a Cross-Coupled Quadrature Harmonic Oscillator
}

\author{
Torsten Djurhuus, Viktor Krozer, Senior Member, IEEE, Jens Vidkjær, Member, IEEE, and Tom K. Johansen
}

\begin{abstract}
The dynamic equations governing the cross-coupled quadrature harmonic oscillator are derived assuming quasi-sinusoidal operation. This allows for an investigation of the previously reported tradeoff between close-to-carrier phase noise and quadrature precision. The results explain how nonlinearity in the coupling transconductances, in conjunction with a finite amplitude relaxation time and de-tuning of the individual oscillators, cause close-to-carrier AM-to-PM noise conversion. A discussion is presented of how the theoretic results translate into design rules for quadrature oscillator ICs. SPECTRE RF simulations verify the developed theory.
\end{abstract}

Index Terms-AM-to-PM noise conversion, $L C$ oscillator, nonlinear analysis, phase error, phase noise, quadrature oscillator.

\section{INTRODUCTION}

Q UADRATURE signals are an essential part of modern RF-communication architectures such as zero-IF and image reject receivers. Over the years, several circuits producing such signals have been proposed. Examples include a frequency division scheme and polyphase filters.

$L C$ oscillators coupled unilaterally in a ring structure [1]-[6] have proven to be a popular alternative, producing multiphase signals without excessive noise or power dissipation penalties. In this paper, we shall consider the coupling of two $L C$ differential oscillators in a ring structure with separate bias for the coupling network as reported in [3]. This system is also known as a cross-coupled quadrature oscillator. It delivers two equal amplitude signals that are ideally $90^{\circ}$ out of phase. However, any asymmetry present in the circuit will result in a deviation from this ideal.

Various forms of linear and graphical analysis of this circuit has been attempted before [4], [6]-[9], but a qualitative model, which includes circuit parameters and nonlinearities, is still missing.

The paper is organized as follows. In Section II, a quick introduction to the modeling of circuit nonlinearities will be given. Section III contains the derivation of a quasi-sinusoidal dynamic model pertaining to the cross-coupled quadrature harmonic oscillator. Section IV explores the consequence of asymmetries in the oscillator and coupling blocks, while Section V concerns the spectrum of the noise perturbed oscillator. The theory outlined here allows the following to be established.

Manuscript received October 22, 2004; revised March 1, 2005. This paper was recommended by Associate Editor I. M. Filanovsky.

The authors are with the Department of Electromagnetic Systems, Ørsted-DTU, Technical University of Denmark, 2800 Lyngby, Denmark (e-mail: tdj@oersted.dtu.dk).

Digital Object Identifier 10.1109/TCSI.2005.853586
1) AM-to-PM noise conversion is significant and cannot be ignored, contrary to what is normally assumed for bilaterally coupled oscillators [10].

2) AM-PM close-to-carrier phase noise is a consequence of a nonlinear coupling transconductance and a finite oscillator amplitude relaxation time.

The developed theory gives new insight into the nature of RF quadrature oscillators perturbed by thermal and shot noise sources. Section VI investigates how these theoretical results are employed in the design of oscillator ICs. Finally, Section VII gives a short summation of the main results.

\section{ModELING NONLINEARITY}

A memoryless third-order characteristic is often used as a model of more complex circuit nonlinearities, because it allows a relatively simple analysis. Here, it will be employed to model both the oscillator negative conductance as well as saturation and compression effects in the coupling transconductances. The oscillator block is also known as a van der Pol unit.

The instantaneous output current $I$ is given as

$$
\begin{gathered}
I_{x, i}=g_{m x, i} V_{i}-\beta_{x, i} V_{i}^{3}=g_{m x, i}\left(V_{i}-K_{x, i} V_{i}^{3}\right), \\
x \in\{o, c\} ; \quad i \in\{1,2\}
\end{gathered}
$$

where $V$ is the instantaneous input voltage. The subscript $x$ refers to the oscillator or coupling transconductance and $i$ to the node number in Fig. 1. ${ }^{1}$ Normally, the above-mentioned characteristic will be implemented by a transistor differential pair, as is indicated in Fig. 1. This circuit block is characterized through its low-frequency transconductance function, which in the case of bipolar transistors is

$$
I=\frac{I_{\mathrm{ET}}}{2} \tanh \left(\frac{V}{2 V_{t}}\right)
$$

where $I_{\mathrm{ET}}$ is the tail current source of the pair and $V_{t}$ is the thermal voltage. Equation (1) can also be specified by a constant $V_{\text {ref, }}$, which defines where the slope changes sign

$$
V_{\text {refx,i }}=\sqrt{\frac{1}{3 K_{x, i}}}, \quad x \in\{o, c\} ; \quad i \in\{1,2\} .
$$

When we in the following talk about how nonlinear a characteristic is, we refer to the location of $V_{\text {ref }}$ on the voltage axis. The gain of the characteristic is controlled by $g_{m}$, which can be adjusted independently of $V_{\text {ref }}$ or $K$.

\footnotetext{
${ }^{1}$ Throughout this paper, we shall use the subscripts $\{o, c\}$ to distinguish between the oscillators and coupling transconductors and the subscripts $\{i, j\}$ to denote the node number. An example would be $X_{c, i}$, where the variable $X$ belongs to the coupling transconductor at node $i$.
} 


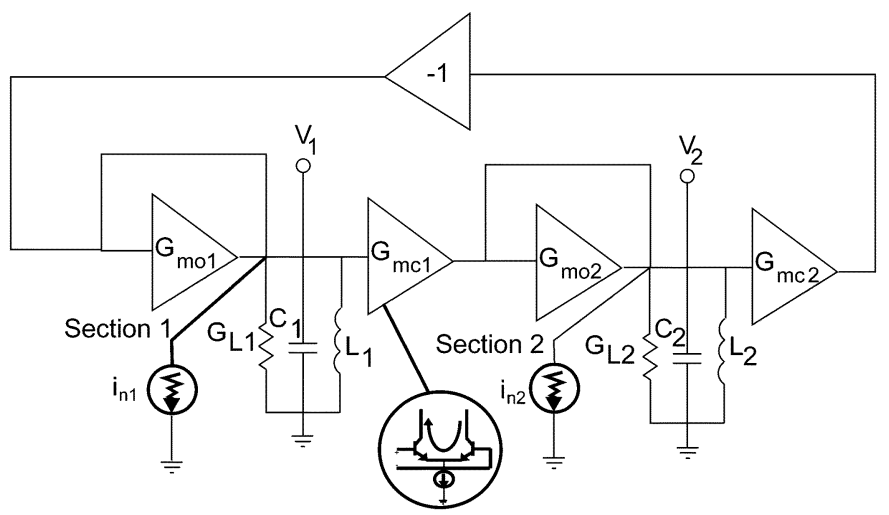

Fig. 1. Cross-coupled quadrature harmonic oscillator single-ended block diagram. The gain blocks are implemented by a transistor differential pair. $G_{m o}$ and $G_{m c}$ are the oscillator negative conductance and coupling transconductance, respectively. The top triangle symbolizes the $180^{\circ}$ phase shift of the cross coupling. Currents $i_{n 1}$ and $i_{n 2}$ represent resonator thermal noise.

The model described in this paper is quasi-sinusoidal, implying a sinusoidal carrier solution. The limitations on the different parameters are revealed by considering the original van der Pol oscillator [11]

$$
\frac{g_{m o}-G_{L}}{G_{L}} \frac{1}{Q}=\frac{\mu_{o}}{Q} \lesssim 0.1
$$

where $G_{L}$ is the resonator conductance as depicted in Fig. 1. Under the above condition, the carrier can be assumed to be sinusoidal without any great loss of accuracy. The different nonlinearity blocks are then approximated by their first harmonic describing function. Inserting the input voltages $V_{i}=A_{i} \cos \left(\omega t+\phi_{i}\right)$ into (1), the first harmonic transconductance is defined as

$$
G_{m x, i}^{1}=g_{m x, i}-\frac{3}{4} \beta_{x, i} A_{i}^{2}, \quad x \in\{o, c\} ; \quad i \in\{1,2\} .
$$

The superscript signifies that only the first harmonic is considered. In order to simplify notation, this shall be omitted in the following, and (5) is referred to as the (large-signal) oscillator or coupling transconductance.

The parameter $\mu_{o}$, defined in (4), is related to the amplitude relaxation time of the oscillator. Differentiating (5) with respect to the amplitude and using the expression for the free running amplitude of a van der Pol oscillator, one can derive the expression

$$
\mu_{o}=-\frac{1}{2} \frac{A}{G_{L}} \frac{d G_{m o}}{d A}=\frac{s}{2} .
$$

Here, $s$ is the amplitude saturation factor introduced in the classical paper [12] by Edson. The amplitude relaxation time $\tau_{o}$ of the oscillator is then given as

$$
\tau_{o}=\frac{Q}{s \pi} T_{0}=\frac{Q}{2 \pi \mu_{o}} T_{0}
$$

where $T_{0}$ is the oscillation period. Equation (6) relates $\mu_{o}$ to different configurations of the oscillator energy restoring circuit (active part) which is normally implemented using a cross-coupled differential transistor topology with the low-frequency characteristic in (2). Assuming no parasitic capacitance at the common emitter node and instantaneous switching of the transistors, the first harmonic transconductance is well approximated by $G_{m o}=2 I /(A \pi)$. Using (6), it is then found that $\mu_{0}=0.5$ for this type of oscillator. The cross-coupled differential pair, assuming instantaneous switching, works as an ideal limiter producing a square-wave current. It is clear that the ideal limiter must have the best amplitude suppression characteristic possible with this topology and therefore the lowest amplitude relaxation time. The conclusion is therefore that any quadrature oscillator based on the cross-coupled pair topology must have a $\mu_{o}$ in the range $[0 ; 0.5]$.

\section{QUASI-SinusoIDAl DyNAMIC Model}

Referring to the block diagram in Fig. $1,{ }^{2}$ an out-of-phase current is injected into each section by coupling transconductances $G_{m c, 1}$ and $G_{m c, 2}$. The operating frequency $\omega_{1}$ can be related to the resonator tank phase shift $\psi$ through

$$
\frac{\omega_{1}}{\omega_{0}}= \pm \frac{\tan (\psi)}{2 Q}+\frac{1}{2 Q} \sqrt{4 Q^{2}+\tan ^{2}(\psi)}
$$

where $\omega_{0}$ is the resonator natural frequency and $Q$ is the loaded quality factor. If $Q \geq 5$ and $\psi \leq 70^{\circ}$, no significant accuracy is lost by the approximation

$$
\frac{\omega_{1}}{\omega_{0}} \approx \pm \frac{\tan (\psi)}{2 Q}+1 \Leftrightarrow \frac{\tan (\psi) \omega_{0}}{2 Q} \approx \mp\left(\omega_{1}-\omega_{0}\right)=\mp \Delta \omega .
$$

As will be shown later, the strength of coupling is related to the resonator phase shift. In the following, the terms coupling range and tuning range will be taken to mean the same thing as no external frequency control (i.e., varactors) is included in the model discussed here. Linearizing the resonator admittance around the new operating frequency in (8) yields the following narrow-band approximation:

$$
\begin{aligned}
Y_{i} \approx & G_{L i}\left(1+j \tan \left(\psi_{i}\right)\right) \\
& +j G_{L i} \sqrt{4 Q_{i}^{2}+\tan ^{2}\left(\psi_{i}\right)} \frac{\left(\omega-\omega_{1}\right)}{\omega_{1}}, \quad i \in\{1,2\}
\end{aligned}
$$

where $\omega$ is the instantaneous operating frequency. Node voltages in Fig. 1 are assumed to have the form $V_{1}=$ $\Re\left\{A_{1} e^{j\left(\omega_{1} t+\phi_{1}\right)}\right\}$ and $V_{2}=\Re\left\{j A_{2} e^{j\left(\omega_{1} t+\phi_{2}\right)}\right\}$. It is important to note that any amplitude or phase transients are slowly varying functions. This means that we can consider them constant over one period of the carrier. The quasi-static behavior of the oscillator envelope and phase implies that the resonator quality factor is much greater than one. The dynamic equations of the system in Fig. 1 are developed in the following steps (see Appendix I or [7] or [10] for details).

Step 1) A phasor equation for each of the two nodes is derived using the function in (10) together with Kurokawa's substitution [13]: $\omega \rightarrow \omega_{1}-j(1 / A)(d A / d t)+(d \phi / d t)$.

Step 2) Orthogonal contributions are separated by multiplying each side of all equations by either $\cos \left(\omega_{1} t+\right.$ $\left.\phi_{i}\right)$ or $\sin \left(\omega_{1} t+\phi_{i}\right)$, respectively. The resulting equations will contain dc terms, including $A_{i}$ and $\phi_{i}$, and fast-varying terms at higher harmonics.

${ }^{2}$ Here, we consider the single-ended equivalent of the actual circuit as depicted in Fig. 1. For an example of a real implementation, we refer to [3], where a complete schematic is included. 
Step 3) The dc terms are isolated by averaging the equations over one period of the carrier.

This leads to a set of coupled differential equations describing the amplitude and phase dynamics of the system

$$
\begin{gathered}
\frac{d A_{i}}{d \tau_{i}}=\mu_{o, i}\left[1-\left(\frac{A_{i}}{\alpha_{i}}\right)^{2}\right] A_{i}-\frac{G_{m c, j}}{G_{L i}} \sin \left(\phi_{j}-\phi_{i}\right) A_{j} \\
\frac{d \phi_{i}}{d \tau_{i}}=\frac{2 Q_{i}}{\omega_{0 i}} \Delta \omega_{i}+\frac{G_{m c, j}}{G_{L i}} \cos \left(\phi_{j}-\phi_{i}\right) \frac{A_{j}}{A_{i}} \\
\quad(i, j) \in\{1,2\} ; \quad i \neq j
\end{gathered}
$$

where $G_{m c, j}$ is the large-signal coupling transconductance as defined in (5), $\mu_{o, i}$ follows from (4), and $\alpha_{i}=$ $\sqrt{4\left(g_{m o, i}-G_{L i}\right) /\left(3 \beta_{o, i}\right)}$ is the free running amplitude, i.e., the amplitude of an uncoupled oscillator. We have also used the time normalization $\tau_{i}=t \cdot \omega_{3 \mathrm{~dB}, i}$ with $\omega_{3 \mathrm{~dB}, i}=\omega_{1} / \sqrt{4 Q_{i}^{2}+\tan ^{2}\left(\psi_{i}\right)} \approx \omega_{1} /\left(2 Q_{i}\right)$.

\section{EFFect of Asymmetry on QUADRATURe Precision}

Any asymmetry in the coupling circuits or the individual oscillators will result in a steady-state that differs from the ideal. As the solution is assumed sinusoidal, the steady state is given by $V_{i}=\hat{A}_{i} \cos \left(\omega_{1} t+\hat{\phi}_{i}\right)$, where hats denote a constant steadystate amplitude and phase offset. Setting the left-hand side in (11) and (12) equal to zero, and using (9), we have

$$
\begin{aligned}
\mu_{o, i}\left[1-\left(\frac{\hat{A}_{i}}{\alpha_{i}}\right)^{2}\right] \hat{A}_{i}= & \mp \frac{\widehat{G}_{m c, j}}{G_{L i}} \sin (\Delta \hat{\phi}) \hat{A}_{j} \\
\tan \left(\psi_{i}\right)= & \frac{\widehat{G}_{m c, j}}{G_{L i}} \cos (\Delta \hat{\phi}) \frac{\hat{A}_{j}}{\hat{A}_{i}} \\
& (i, j) \in\{1,2\} ; \quad i \neq j
\end{aligned}
$$

where $\Delta \hat{\phi}=\hat{\phi}_{1}-\hat{\phi}_{2}$ and $\widehat{G}_{m c, j}=g_{m c, j}-3 / 4 \beta_{c, j} \hat{A}_{j}^{2}$. We are interested in investigating how asymmetry in the resonators and active blocks (see Fig. 1) affects quadrature precision. The scope is limited to the case where nonlinearities of the active blocks are equal from one section to the next (i.e., $K_{c, 1}=K_{c, 2}=K_{c}$ and $K_{o, 1}=K_{o, 2}=K_{o}$ ).

In the following, it is assumed that $\hat{A}_{2} / \hat{A}_{1}=1+\varepsilon$, $g_{m c, 1} / g_{m c, 2}=1+\eta, \alpha_{1} / \alpha_{2}=1+\nu, L_{1} / L_{2}=1+\epsilon_{l}$, $C_{1} / C_{2}=1+\epsilon_{c}, G_{L 1} / G_{L 2}=1+\epsilon_{g}$ with $|\varepsilon|,|\eta|,|\nu|,\left|\epsilon_{l}\right|,\left|\epsilon_{c}\right|$, $\left|\epsilon_{g}\right| \ll 1$. In Appendix II, it is shown how (13) and (14) can be manipulated into the following expressions:

$$
\begin{aligned}
\varepsilon & \approx \frac{Q\left(\epsilon_{l}+\epsilon_{c}\right)}{2(1-\lambda) \zeta}+\frac{\eta}{2(1-\lambda)} \\
\sin (\Delta \hat{\phi}) & \approx-\frac{\mu_{o}}{1-\lambda} \frac{\left(\epsilon_{l}+\epsilon_{c}\right) Q}{2 \zeta^{2}}-\frac{\mu_{o}}{1-\lambda} \frac{\eta}{2 \zeta}-\mu_{o} \frac{\nu}{\zeta}
\end{aligned}
$$

where $\zeta=\widehat{G}_{m c} / G_{L}=\tan (\psi)$ denotes the coupling strength as derived from (14). The parameter $\lambda$ is defined in (35) of Appendix II. The inverse quadratic dependence on the coupling strength in the first term of (16) was first derived in [6] using graphical phasor analysis. Note that the effect of conductor asymmetry is contained implicitly through $\nu$.
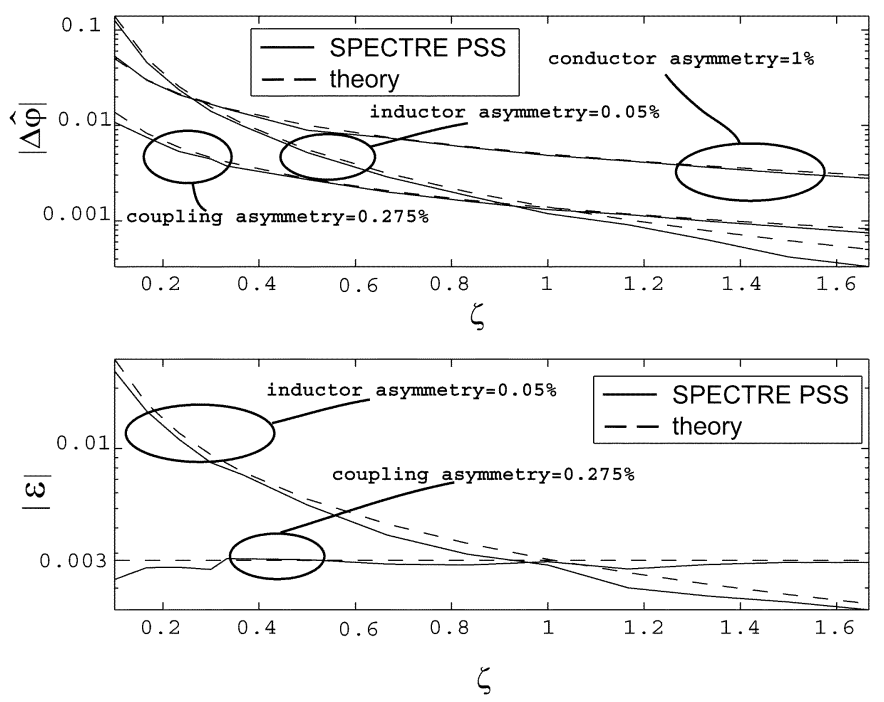

Fig. 2. Phase and amplitude error $\left(\varepsilon=\hat{A}_{2} / \hat{A}_{1}-1\right)$ as a result of small mismatches $\left(\epsilon_{l}=0.05 \%, \epsilon_{g}=1 \%\right.$, and $\left.\eta=0.275 \%\right)$ in the two resonators and coupling sections. Simulations are for van der Pol oscillators coupled through third-order nonlinearities. Top: Phase error (in radians). Equation (16) $=$ broken line, SPECTRE PSS simulation = solid line. Bottom: Amplitude error. Equation $(15)=$ broken line, SPECTRE PSS simulation $=$ solid line.

Equations (15) and (16) are compared with SPECTRE Periodic Steady State (PSS) simulations in Fig. 2 with $\epsilon_{l}=0.05 \%$, $\epsilon_{g}=1 \%$, and $\eta=0.275 \%$. It is important to note that simulations are performed on van der Pol oscillators with ideal thirdorder coupling sections. Simulations containing SiGe BiCMOS components are included in Sections V and VI.

From Fig. 2 and (15) and (16), it follows that the phase error dominates for lower couplings. For $\zeta>\mu_{o},{ }^{3}$ the amplitude error is more significant. This is an important result as amplitude and phase error degrade the image rejection equally when the oscillator is integrated in a receiver structure. However, the amplitude imbalance can easily be removed by following the oscillator by a limiting buffer. Another interesting point, which can be extracted from (15) and (16), is that asymmetry in the passive parts of the resonator is the main source of amplitude and phase error. This is because they enter the equations multiplied by a factor $Q$.

\section{Spectrum of a Noise-Perturbed Oscillator}

Amplitude and phase noise in oscillators originate from perturbations of the steady-state by small noise-like signals. The amplitude and phase of the system can then be written as $A_{i}=$ $\hat{A}_{i}+\delta A_{i}$ and $\phi_{i}=\hat{\phi}_{i}+\delta \phi_{i}$, where $\delta A_{i}$ and $\delta \phi_{i}$ are transient amplitude and phase responses of the oscillator. These variables are considered to be quasi-static as the resonator is assumed to have a high $Q$ [7].

The noise currents $i_{n}$ in parallel with the resonator tanks in Fig. 1 represent resonator thermal noise. Mixing effects between the carrier and noise signals, as well as internal noise sources in the oscillator active part and coupling transconductances, are not part of the theory discussed in this paper. However, the model can be augmented by employing a noise factor calculated

\footnotetext{
${ }^{3}$ Here, $\mu_{o}=0.5$ is used. In Section II, it is shown that this value of $\mu_{o}$ corresponds to a cross-coupled pair working as an ideal limiter.
} 
using large-signal small-signal analysis [14], [6]. With mixing effects being ignored, the noise can be modeled by a stationary narrow-band Gaussian noise process

$$
i_{n, i}=i_{n c, i} \cos \left(\omega_{1} t\right)-i_{n s, i} \sin \left(\omega_{1} t\right), \quad i \in\{1,2\}
$$

where $i_{n c}$ and $i_{n s}$ are slowly varying uncorrelated stationary Gaussian processes. Linearizing the set of (11) and (12) around a steady-state carrier with the noise currents in (17) included and assuming full symmetry $\left(G_{L 1}=G_{L 2}=G_{L}\right.$, $\widehat{G}_{m c, 1}=\widehat{G}_{m c, 1}=\widehat{G}_{m c}$ etc.), the following set of linear first-order coupled differential equations, pertaining to the noise induced quasi-static transients, can be derived:

$$
\begin{aligned}
\frac{1}{\hat{A}} \frac{d \delta A_{i}}{d \tau}= & \mu_{o}\left[1-3\left(\frac{\hat{A}}{\alpha}\right)^{2}\right] \frac{\delta A_{i}}{\hat{A}}-\frac{\widehat{G}_{m c}}{G_{L}}\left(\delta \phi_{j}-\delta \phi_{i}\right)-G_{n i} \\
\frac{d \delta \phi_{i}}{d \tau}= & \frac{\widehat{G}_{m c}}{G_{L}}\left(1-2 \mu_{c}\right) \frac{\delta A_{j}}{\hat{A}}-\frac{\widehat{G}_{m c}}{G_{L}} \frac{\delta A_{i}}{\hat{A}}-B_{n i} \\
& (i, j) \in\{1,2\} ; \quad i \neq j
\end{aligned}
$$

where $G_{n}$ and $B_{n}$ are stationary Gaussian noise processes known as the normalized noise conductance and susceptance, respectively [10], and

$$
\mu_{c}=\frac{g_{m c}-\widehat{G}_{m c}}{\widehat{G}_{m c}}
$$

We have also used a linearized version of $G_{m c} A$ [see Section II, (5)]

$$
\begin{aligned}
g_{m c} \delta A-\frac{9}{4} \beta_{c} \hat{A}^{2} \delta A & =\widehat{G}_{m c} \delta A\left(1-\frac{\frac{3}{2} \beta_{c} \hat{A}^{2}}{\widehat{G}_{m c}}\right) \\
& =\widehat{G}_{m c} \delta A\left(1-\frac{2\left(g_{m c}-\widehat{G}_{m c}\right)}{\widehat{G}_{m c}}\right) \\
& =\widehat{G}_{m c} \delta A\left(1-2 \mu_{c}\right) .
\end{aligned}
$$

In Appendix III, the spectrum of the noise-perturbed oscillator is found by Fourier transforming the time-domain representation in (18) and (19). Close to the carrier, this results in the following expressions:

$$
\begin{aligned}
& \lim _{\omega_{m} \rightarrow 0}\left\langle\widetilde{\delta A^{2}}\right\rangle=\frac{\frac{N_{0}}{P_{0}}}{2\left(\frac{\widehat{G}_{m c}}{G_{L}}\right)^{2}\left(1-\mu_{c}\right)^{2}} \times\left\{\frac{\left(\frac{\widehat{G}_{m c}}{G_{L}}\right)^{2}+\mu_{o}^{2}}{4 \mu_{o}^{2}}\right\} \\
& \lim _{\omega_{m} \rightarrow 0}\left\langle\widetilde{\delta \phi^{2}}\right\rangle=\mathfrak{L}_{C}\left(\omega_{m}\right) \\
& =\left[\frac{\omega_{1}}{\sqrt{2} \sqrt{4 Q^{2}+\tan ^{2}(\psi)}}\right]^{2} \\
& \times\left[1+\left(\frac{\mu_{c}}{\mu_{o}}\right)^{2} \tan ^{2}(\psi)\right] \frac{N_{0}}{P_{0} \omega_{m}^{2}}
\end{aligned}
$$

where $\mathfrak{L}_{C}\left(\omega_{m}\right)$ denotes phase noise of the coupled oscillator at offset $\omega_{m}$ and $N_{0} / P_{0}$ is the noise-to-signal power ratio. The $\left(\mu_{c} / \mu_{o} \tan (\psi)\right)^{2}$ term in (23) originates from the $\underline{\underline{N}}_{21}$ transfer matrix, defined in (54) of Appendix III, and therefore represents AM-to-PM noise conversion.

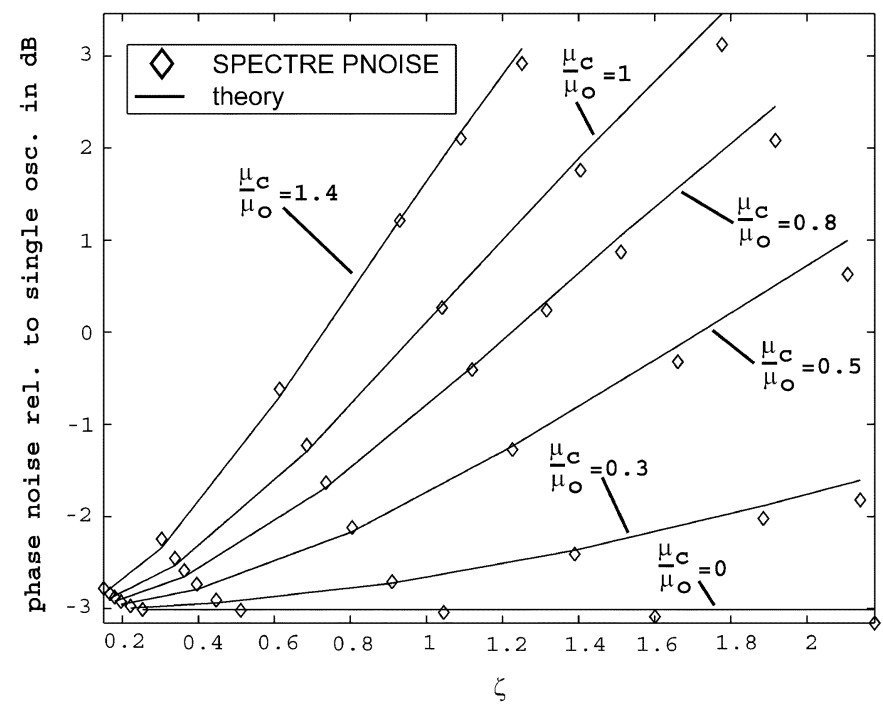

Fig. 3. Figure showing (23) relative to (25) in decibels as a function of the coupling strength $\zeta$ for six different $\mu_{c} / \mu_{o}$ ratios. The simulations are of van der Pol oscillators coupled through a third-order nonlinearity. SPECTRE PNOISE phase noise simulations $=\diamond$, theory $=$ solid line. The different curves are created by fixing $K_{c}$ and, hence, the nonlinearity of the coupling (see Section II) and varying $g_{m c} . \mu_{o}=0.5$ throughout.

Equations (58) and (59) should return to the equivalent equations for the free-running case when the coupling tends toward zero

$$
\begin{aligned}
\lim _{\widehat{\boldsymbol{G}}_{m c}, \omega_{m} \rightarrow 0}\left\langle\widetilde{\delta A^{2}}\right\rangle & =\frac{N_{0}}{4 \mu_{O}^{2} P_{0}} \\
\lim _{\widehat{\boldsymbol{G}}_{m c}, \omega_{m} \rightarrow 0}\left\langle\widetilde{\delta \phi^{2}}\right\rangle & =\mathfrak{L}\left(\omega_{m}\right)=\left(\frac{\omega_{0}}{2 Q}\right)^{2} \frac{N_{0}}{P_{0} \omega_{m}^{2}}
\end{aligned}
$$

where it is used that

$$
\begin{aligned}
\lim _{\widehat{G}_{m c} \rightarrow 0} \omega_{1} & =\omega_{0} \\
\lim _{m c} \omega_{3 \mathrm{~dB}} & =\frac{\omega_{0}}{2 Q} .
\end{aligned}
$$

The results in (24) and (25) agree with those found in the literature [10], [15].

Equation (23), relative to (25), is plotted in Fig. 3 together with a series of SPECTRE PNOISE simulations for six different $\mu_{c} / \mu_{o}$ ratios. The SPECTRE simulations are done on van der Pol oscillators coupled through third-order voltage-controlled current sources. In Fig. 3, we consider phase noise at a fixed offset for a cross-coupled quadrature oscillator (see Fig. 1) and an equivalent single oscillator unit. Fig. 3 results when these two values, in decibels, are subtracted and further normalized for the different operating frequencies. ${ }^{4}$ The different curves in Fig. 3 are created by fixing $K_{c}$ and then varying $g_{m c}$. The parameters $\mu_{o}$ and $K_{o}$ are held constant throughout. The nonlinearity of the coupling sections is determined by the $V_{\text {ref }}$ parameter and hence by $K_{c}$ as illustrated in (3). From Fig. 3, it is seen that, as the coupling strength increases, AM-PM conversion deteriorates

${ }^{4}$ From (8), it is seen that the frequency changes with the coupling strength. As phase noise is proportional to the operating frequency squared [see (23) and (25)], normalization is achieved by including the factor $20 \log \left(\omega_{1} / \omega_{0}\right)$. 


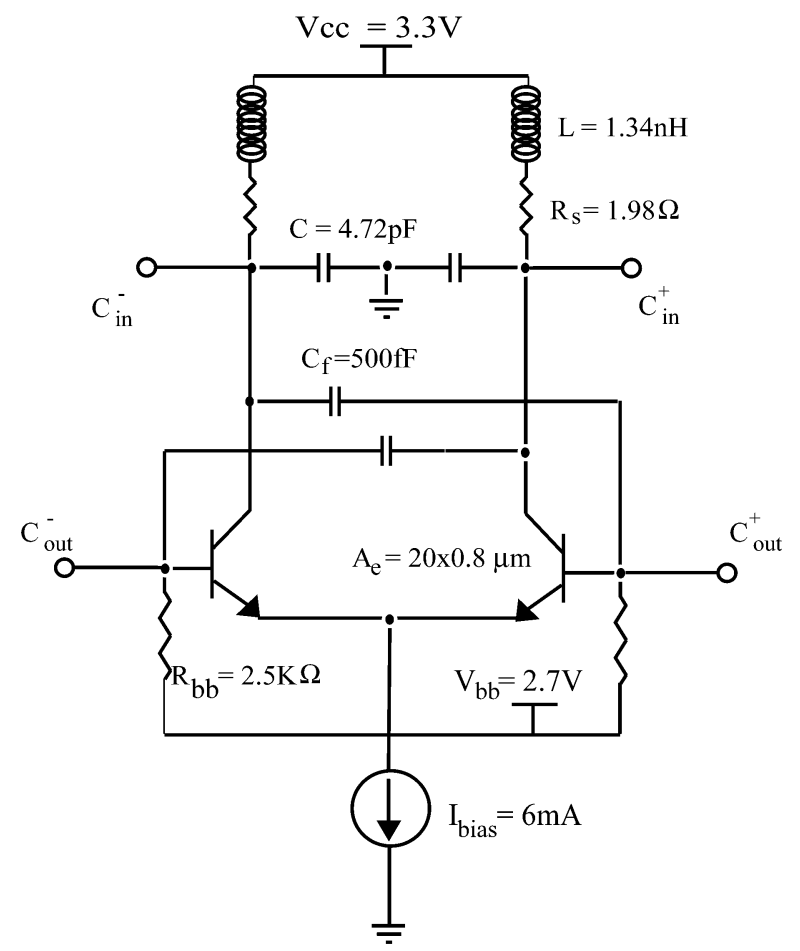

Fig. 4. A 2-GHz $0.8-\mu \mathrm{m} \mathrm{SiGe} \mathrm{differential} L C$ oscillator implemented using a cross-coupled pair topology. Feedback capacitors $C_{f}$ are included to keep the cross-coupled pair in the linear region. The four ports $C_{\text {in }}^{ \pm}, C_{\text {out }}^{ \pm}$are the coupling input and output ports. By this, it is meant that the output ports of the oscillator should be connected to the input ports of the coupling circuit.

the overall noise performance of the oscillator. However, from (15) and (16), we see that effects of circuit asymmetries are reduced through stronger coupling. It then follows that there exists a tradeoff between phase noise and quadrature precision in a cross-coupled quadrature harmonic oscillator [5], [6]. Another interesting fact, which can be extracted from Fig. 3 and (23), is that the coupling transconductance nonlinearity impacts on the level of AM-to-PM conversion. By decreasing the nonlinearity of these blocks, (23) predicts that the close-to-carrier AM-PM conversion can be completely cancelled. This novel result can only be found through nonlinear analysis, and it represents the main contribution of this paper. Finally, it is noted from (23) that the AM-PM noise conversion is proportional to the square of the oscillator amplitude relaxation time [16],[17], as represented by the parameter $1 / \mu_{o}^{2}$ [see (7)]. This intuitively pleasing result is a characteristic of most $L C$ oscillators. The above results illustrate that a highly tunable quadrature oscillator could be created, with low phase noise over the entire tuning range, if one could find a way to minimize the product of the coupling nonlinearity and the oscillator amplitude relaxation time. Application of these new insights to practical RF ICs is discussed in Section VI.

Fig. 4 shows the schematic of a $2-\mathrm{GHz}$ differential $L C$ transistor oscillator that will be used in the following simulations. The transistors are taken from $0.8-\mu \mathrm{m} \mathrm{SiGe} \mathrm{technology} \mathrm{and} \mathrm{the}$ series $L-R$ circuit is a model of an inductor from the same process. The bias network, a simple current mirror, is not shown. Varactors are omitted in order to avoid confusion between the AM-PM noise conversion due to the nonlinear $C-V$ characteristic and the AM-PM conversion described here. The differential amplitude is $1 V_{p}$ and the $Q$ of the inductor is 8.5 at $2 \mathrm{GHz}$. The phase noise of the single oscillator is $-113.3 \mathrm{dBc}$ at $1-\mathrm{MHz}$ offset. Two important alterations occur when we move from the simple model to a real implementation.

1) The resonator is built with lossy inductors which determine the $Q$ of the circuit.

2) The parameters $\mu_{o}$ and $K_{o}$ are no longer specifically defined and have to be estimated.

As the resonator is implemented with lossy inductors, the $Q$-factor and oscillation amplitude is a function of the operating frequency. Consequently, it is necessary to normalize for the $Q$ and the amplitude, as well as for the frequency. The estimate $\mu_{o} \approx 0.28$ was found to produce the best fit. $K_{o}$ can be estimated using standard formulas for the third-order harmonic distortion of an $L C$ oscillator. Using this approach to obtain a first guess, the estimate $K_{o} \approx 1.57$ was found to produce an acceptable fit.

In Fig. 5(a) and (b), we repeat the asymmetry and phase noise simulations of Figs. 2 and 3 with the oscillator block shown in Fig. 4. The coupling transconductances are implemented by ideal third-order voltage-controlled current sources as described by (1). The phase noise simulations in Fig. 5(a) are conducted at a frequency offset of $1 \mathrm{MHz}$ which ensures that only the $1 / f^{2}$ part of the spectrum is considered. The fact that the amplitude is no longer constant with coupling means that the $\mu_{c}$ parameter changes as the two oscillators are coupled harder. However, the spread is not very large and the parameter is well represented by its average. This is the value included in Fig. 5(a). In Fig. 5(b), the results of a small capacitor asymmetry $\epsilon_{c}=0.1 \%$ are plotted for two different $\mu_{c} / \mu_{o}$ ratios. The maximum deviation between theory and simulation is below $0.28^{\circ}$ for the phase and $1.4 \times 10^{-2}$ for the amplitude. Fig. 5(a) and (b) illustrates that the complexities of the oscillator block in Fig. 4 can be well fitted to the much simpler van der Pol model once the parameters $\mu_{o}$ and $K_{o}$ have been estimated.

In the case where the nonlinearity of coupling and oscillator transconductance are identical, it follows that

$$
\begin{aligned}
\frac{\mu_{c}}{\mu_{o}} & =\frac{\frac{g_{m c}-\widehat{G}_{m c}}{\widehat{G}_{m c}}}{\frac{g_{m}-\widehat{G}_{m o}}{\widehat{G}_{m o}}}=\frac{\widehat{G}_{m o}}{\widehat{G}_{m c}} \frac{\beta_{c}}{\beta_{o}} \\
& =\frac{g_{m o}}{g_{m c}} \frac{1-\frac{3}{4} K_{o} \hat{A}^{2}}{1-\frac{3}{4} K_{c} \hat{A}^{2}} \frac{\beta_{c}}{\beta_{o}}=\frac{g_{m o}}{\beta_{o}} \frac{\beta_{c}}{g_{m c}}=\frac{K_{c}}{K_{o}}=1
\end{aligned}
$$

where the relation $G_{L}=\widehat{G}_{m o}$ has been used. We now consider the case $\mu_{c} / \mu_{o}=1$. Comparing (23) with Leeson's model for phase noise in single oscillators [18], we can define a new $Q$-factor $Q_{C}$ for the cross-coupled quadrature oscillator as

$$
Q_{C}=\frac{1}{\sqrt{2}} \cos (\psi) \sqrt{4 Q^{2}+\tan ^{2}(\psi)} \approx \underbrace{\sqrt{2}}_{\begin{array}{c}
\text { Mutual } \\
\text { Phase } \\
\text { Locking } \\
\text { ocking }
\end{array}} Q \underbrace{\cos (\psi)}_{\begin{array}{c}
\text { AM-PM } \\
\text { Noise } \\
\text { Conversion }
\end{array}} .
$$

It lumps together the effects of mutual phase locking and AM-PM noise conversion. The result in (29) was first found in [4], ${ }^{5}$ where a phase transfer function was derived through linear

${ }^{5}$ In [4], the noise-to-signal ratio is not normalized to the noise of a single oscillator. This is why the equivalent $Q_{2}$ factor in this paper includes a factor of 2 instead of a factor $\sqrt{2}$. Normalized to the same noise-to-signal ratio, the two results are identical. 


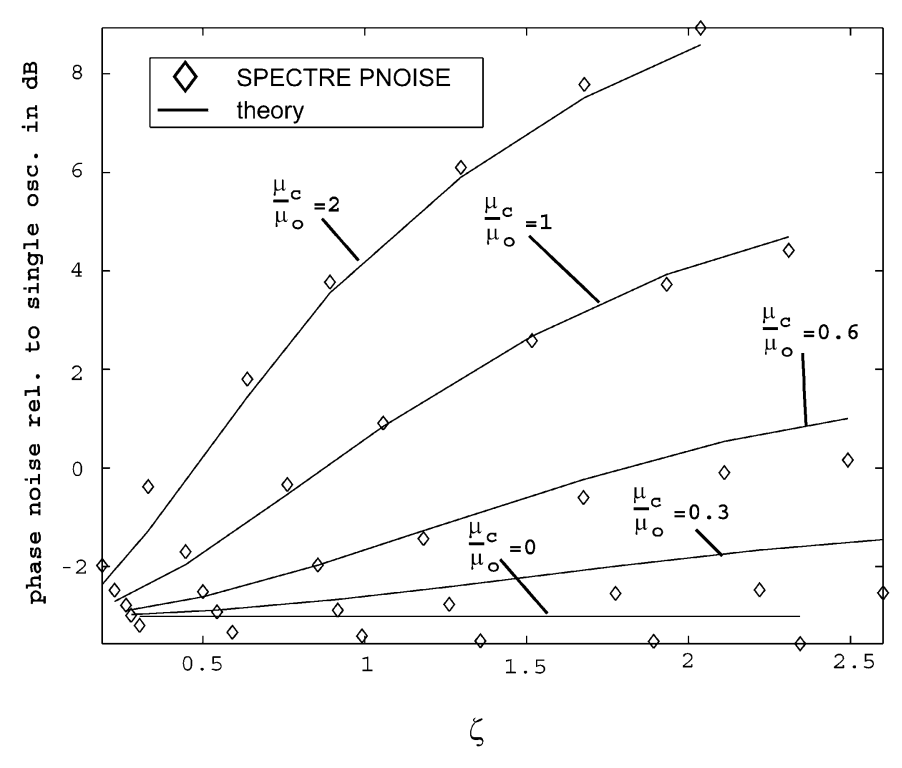

(a)

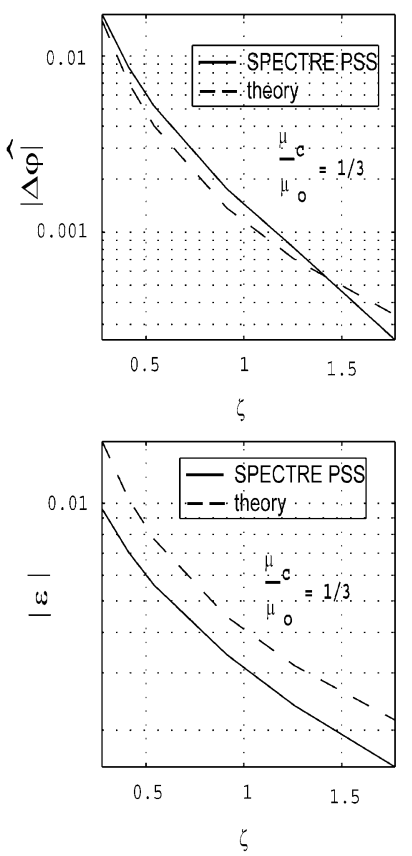

(b)
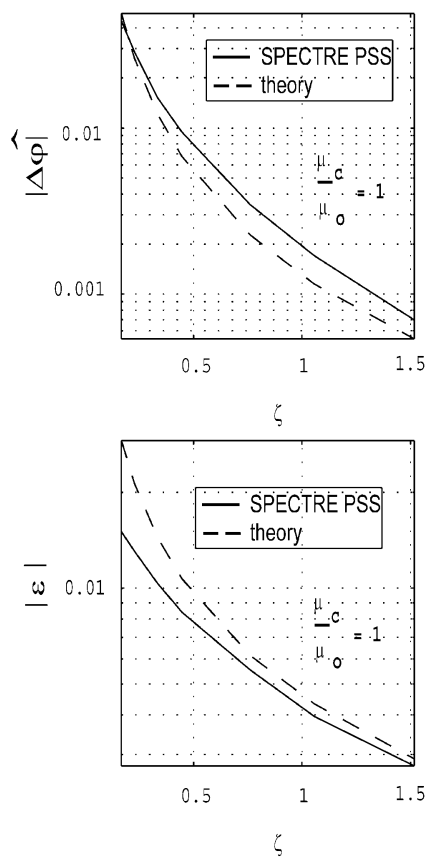

b)

Fig. 5. SPECTRE PSS and PNOISE simulations of a 2-GHz 1- $V_{p} L C$ cross-coupled quadrature oscillator. The oscillator blocks are shown in Fig. 4. The parameter $\mu_{o}$ is determined to be 0.28 and the parameter $K_{o}$ is 1.57 . The coupling transconductance blocks are implemented by ideal third-order voltage-controlled current sources. (a) SPECTRE PNOISE phase noise simulations at 1-MHz frequency offset are shown by $\diamond$ and theory by the solid line. (b) Phase (in radians) and amplitude

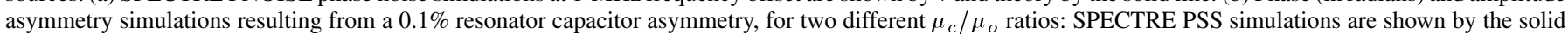
line and theory by the broken line.

analysis. Later, the authors of [6] also derived it using graphical phasor analysis. In [4], it was found that phase shifters inserted in between the oscillator sections were needed in order to make noise and quadrature orthogonal entities. This statement still holds. However, the nonlinear analysis detailed in this paper reveals a completely new aspect. Inspecting (23), we see that introducing linear coupling presents yet another way of eliminating the noise/quadrature tradeoff.

\section{DESIGN CONSIDERATIONS}

In the previous section, it was illustrated how two differential $L C$ oscillators coupled through a linear unilateral coupling network would maintain a low phase-noise performance over the entire tuning/coupling range. This novel result opens the door on low phase-noise quadrature oscillators with large tuning range. However, it is well known that for increased linearity the price paid is an increase in noise figure and a decrease of the gain. This precludes the most obvious linearizing techniques, such as emitter degeneration and multitanh topologies, from practical use as coupling sections in a quadrature oscillator. This paper will therefore not discuss the various existing linearizing techniques but instead focus on the effect of the oscillator amplitude relaxation time on the AM-PM noise conversion performance of the oscillator.

A common emitter capacitor is often included in the bias circuit of the oscillators [3]. This capacitor has several purposes. It acts as a noise filter at the second harmonic, thus restricting the amount of noise that is down-converted by the switching differential pair. Second, it forces the oscillator to pulse its current close to the carrier maximum. This can be shown to be optimal with regard to noise [19]. Finally, it increases the amplitude of oscillation. From the discussion in Section II, it is known that the current switching regime of the cross-coupled differential pair results in the shortest possible amplitude relaxation time or, equivalently, the largest possible $\mu_{o}$, for a given resonator. According to the theory developed in Section V, the AM-PM conversion must then increase when the common-emitter node is decoupled by a large capacitor.

The simulations in this section are for the full quadrature oscillator with the 2-GHz oscillator blocks in Fig. 4 and coupling sections are implemented as differential pair transconductors. A schematic illustrating the topology is shown in Fig. 6. The coupling section transistors have the same size as the cross-coupled transistors in Fig. 4. Both oscillator and coupling sections are biased through a simple resistor degenerated current mirror. Oscillator blocks containing a common-emitter capacitor are referred to here as decoupled oscillators while those without are called current-switched. The bias circuit of the coupling sections does not contain a decoupling capacitor. In Fig. 7(a) the AM-PM noise conversion is shown for the complete $2-\mathrm{GHz}$ quadrature oscillator circuit. This plot is generated similarly to Figs. 3 and 5(a). Two curves are shown, one for a decoupled oscillator and one for a current-switched oscillator. The plots show that the AM-PM noise conversion worsens by including a decoupling capacitor as predicted by (23) and the above discussion. In order to compare the noise performance of the two oscillators over the coupling/tuning range, a figure of merit (FOM) is defined as

$$
\mathrm{FOM}=-\mathfrak{L}_{C}\left(\omega_{m}\right)+20 \log \left(\frac{\omega_{1}}{\omega_{m}}\right)-10 \log \left(P_{\text {diss }}\right)
$$

where $P_{\text {diss }}$ is the total dissipated power in milliwatts. In Fig. 7(b), the FOM is shown for the two oscillators compared in Fig. 7(a). For low coupling strengths, the decoupled oscillator 


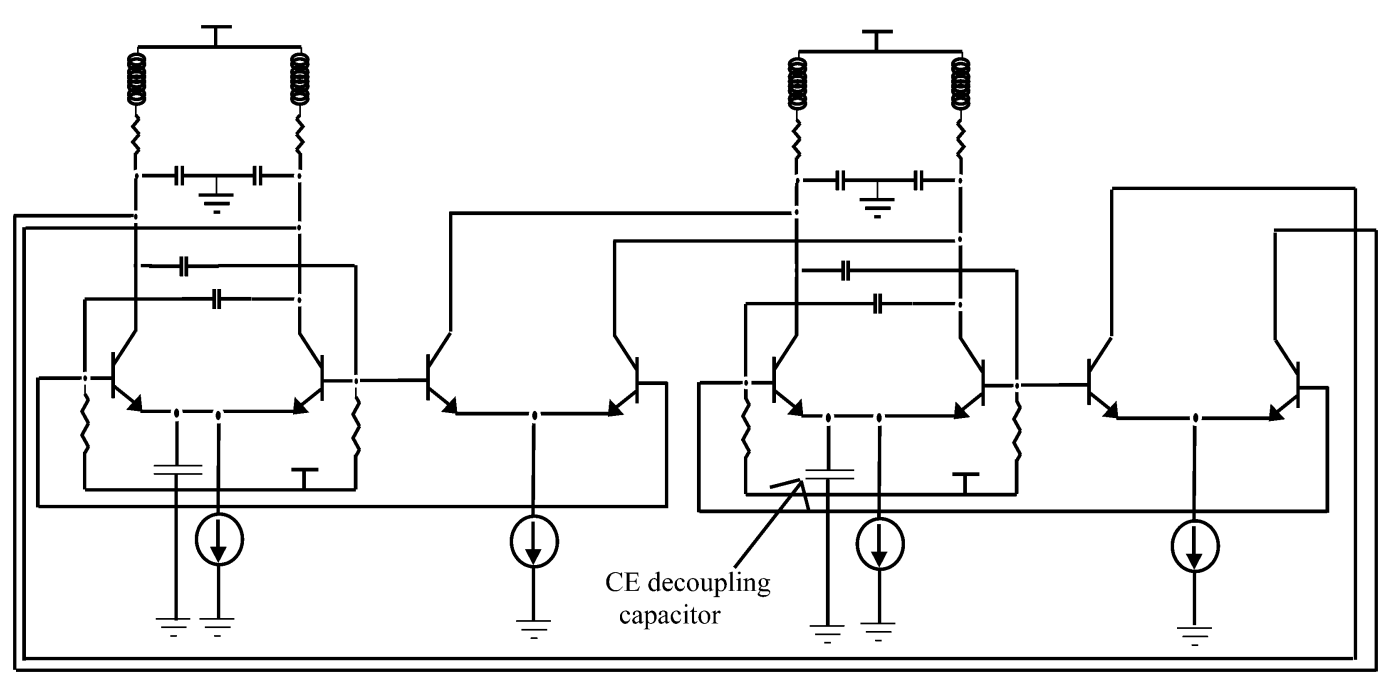

Fig. 6. Full 2-GHz quadrature oscillator. Oscillator blocks are detailed in Fig. 4. The coupling stage transistors have the same size as the oscillator transistors. Oscillators containing a CE capacitor are referred to as decoupled oscillators while those without are known here as current switched oscillators. Current sources are implemented as simple resistor degenerated current mirrors.

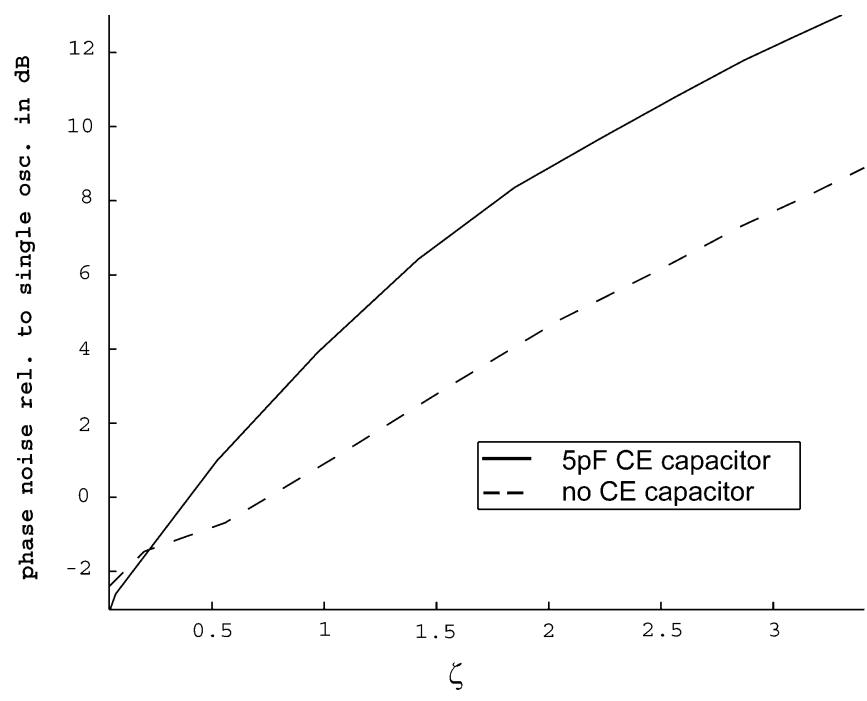

(a)

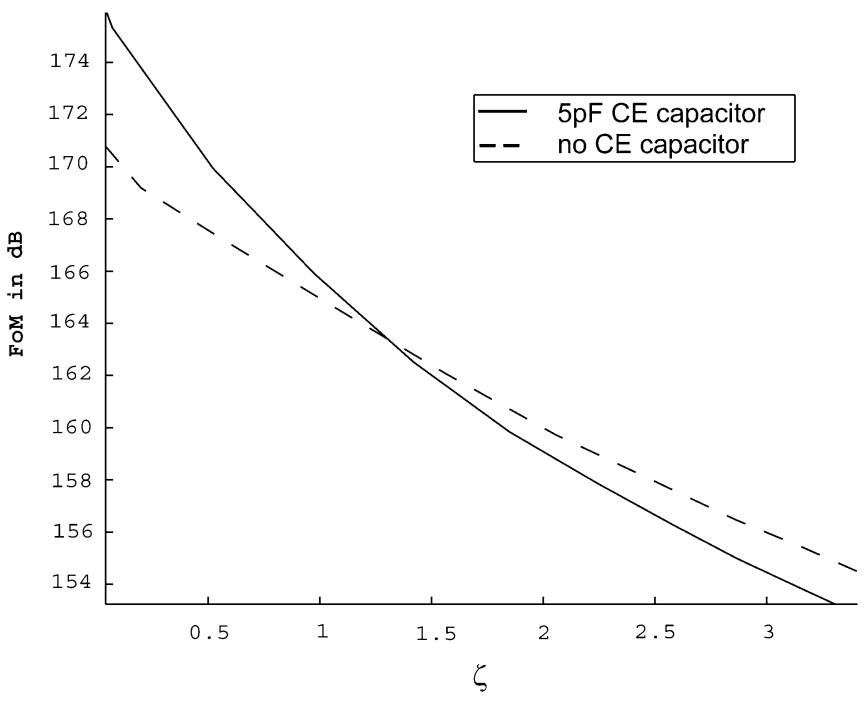

(b)

Fig. 7. SPECTRE PNOISE simulations of a 2-GHz 1- $V_{p} L C$ cross-coupled quadrature oscillator for two different bias configurations. The oscillator blocks are shown in Fig. 4. The coupling transconductance blocks are implemented as differential pair transconductors (see Fig. 6). (a) SPECTRE PNOISE phase noise simulations at $1-\mathrm{MHz}$ frequency offset. The figure shows the relative increase in phase noise (AM-PM contribution + noise from coupling sections) as a function of coupling strength for two different bias configurations. Current-switched oscillator is shown by the broken line and the decoupled oscillator $\left(C_{e}=5 \mathrm{pF}\right)$ is shown by the solid line. (b) SPECTRE PNOISE simulations at 1-MHz frequency offset. The plots show the FOM defined in (30) for the two different configurations compared in (a) as a function of coupling strength.

shows the best FOM as this topology has a superior large-signal noise performance. However, for higher coupling strengths, the difference in AM-PM conversion causes the two curves to move closer to each other, and for coupling strengths above 1.3 the FOM of the current-switched oscillator is actually superior to the decoupled oscillator. This result could not have been predicted, and it shows that the developed theory, however simple, can be applied to complex transistor circuits.

\section{CONCLUSION}

This paper details the derivation of the dynamic equations governing the cross-coupled quadrature harmonic oscillator, including all circuit parameters and nonlinearities. The model is valid under the condition of quasi-sinusoidal operation and quasi-static envelope and phase.

Using the theory developed here, new insight into the effect of noise on the oscillator spectrum was achieved. It was shown how detuning from resonance of the individual oscillators acts together with nonlinearity in the coupling transconductances and a finite amplitude relaxation time to create AM-PM noise conversion. A simple third-order model was shown to be able to fit transistor oscillator circuits after estimating just two constants. The effect of including a common-emitter filter/decoupling capacitor on the noise performance of the quadrature oscillator was discussed. It was shown that the phase noise of the decoupled oscillator deteriorated at higher coupling strengths due to an increased AM-PM close-to-carrier noise conversion. Although the decoupled oscillator configuration had a superior 
noise performance at low coupling strengths, at higher coupling strengths, a configuration without decoupling would perform better.

Previous attempts toward analysis of this oscillator have suggested phase shifters inserted between the sections as one possible way of eliminating the tradeoff between phase noise and quadrature. Using the theory developed in this paper it is seen that an alternative would be to introduce very linear coupling transconductances.

\section{APPENDIX I}

Using (10) and Kurokawas's substitution [10], [13] at node 1 in Fig. 1, one can derive the following phasor equation:

$$
\begin{aligned}
& \left(G_{L 1}-G_{m o, 1}\right) A_{1} e^{j \Phi_{1}}+j G_{L 1} \tan \left(\psi_{1}\right) A_{1} e^{j \Phi_{1}} \\
& \quad-G_{m c, 2} j A_{2} e^{j \Phi_{2}} \\
& +G_{L 1}\left(\frac{1}{A_{1}} \frac{d A_{1}}{d \tau_{1}}+j \frac{d \phi_{1}}{d \tau_{1}}\right) A_{1} e^{j \Phi_{1}}=0
\end{aligned}
$$

with $\Phi_{i}=\omega_{1} t+\phi_{i}$ and $\tau_{1} \approx \omega_{1} /\left(2 Q_{1}\right) t$. Taking the real part of (31), multiplying with $\cos \left(\Phi_{1}\right)$ and $\sin \left(\Phi_{1}\right)$, respectively, and then averaging over one period yields (32) and (33), shown at the bottom of the page, where the relations in (5) and (9) have been used. Repeating the above procedure for section 2 of the circuit in Fig. 1, equivalent expressions can derived for the oscillator at node 2 .

\section{APPENDIX II}

Using (5) from Section II, we have

$$
\begin{aligned}
\frac{\widehat{G}_{m c 1}}{\widehat{G}_{m c 2}} & =\frac{g_{m c 1}}{g_{m c 2}} \frac{1-\frac{3}{4} K_{c} \hat{A}_{1}^{2}}{1-\frac{3}{4} K_{c} \hat{A}_{2}^{2}} \approx(1+\eta) \frac{1-\frac{3}{4} K_{c} \hat{A}_{1}^{2}}{1-\frac{3}{4} K_{c}(1+2 \varepsilon) \hat{A}_{1}^{2}} \\
& \approx(1+\eta)\left(1+\frac{\frac{3}{2} K_{c} \hat{A}_{1}^{2}}{1-\frac{3}{4} K_{c} \hat{A}_{1}^{2}} \varepsilon\right) \\
& \approx(1+\eta)(1+2 \lambda \varepsilon)
\end{aligned}
$$

where it has been assumed that $\hat{A}_{1} \approx \alpha$ and

$$
\lambda=\frac{\frac{K_{c}}{K_{o}} \mu_{o}}{1+\left(1-\frac{K_{c}}{K_{o}}\right) \mu_{o}}
$$

A small deviation between resonator components of the two oscillators can be related to a similar small change in resonator phase shifts. The case where the two inductors differ by a small amount is now examined

$$
L_{1}=L_{2}\left(1+\epsilon_{l}\right) \Leftrightarrow \frac{\omega_{02}}{\omega_{01}} \approx 1+\frac{1}{2} \epsilon_{l}, \quad\left|\epsilon_{l}\right| \ll 1
$$

$$
\frac{\tan \left(\psi_{2}\right)}{\tan \left(\psi_{1}\right)}=1+\kappa, \quad|\kappa| \ll 1 .
$$

The frequency of the oscillators are equal in the steady-state, and (9) yields

$$
\begin{aligned}
\frac{\tan \left(\psi_{2}\right)}{2 R C}+\omega_{02} & =\frac{\tan \left(\psi_{1}\right)}{2 R C}+\omega_{01} \Leftrightarrow \\
\left(\frac{\tan \left(\psi_{2}\right)}{\tan \left(\psi_{1}\right)}-1\right) & =\frac{2 R C\left(\omega_{01}-\omega_{02}\right)}{\tan \left(\psi_{1}\right)} \Leftrightarrow \\
\kappa & =\frac{2 Q_{2}\left(\omega_{01}-\omega_{02}\right)}{\tan \left(\psi_{1}\right) \omega_{02}} \approx-\frac{Q \epsilon_{l}}{\tan (\psi)}
\end{aligned}
$$

where, in the last line, subscripts become superfluous as $\left|\epsilon_{l}\right| \ll$ 1. The above procedure can be repeated for asymmetries in the resonator capacitors and conductors

$$
\kappa \approx-\frac{Q\left(\epsilon_{l}+\epsilon_{c}\right)}{\tan (\psi)}+\epsilon_{g}, \quad\left|\epsilon_{l}\right|,\left|\epsilon_{c}\right|,\left|\epsilon_{g}\right| \ll 1
$$

with $\epsilon_{c}$ and $\epsilon_{g}$ representing capacitor and conductor asymmetry, respectively.

Using (34), (35), and (39), (13) and (14) can be written as

$$
\begin{aligned}
\frac{\hat{A}_{2}}{\hat{A}_{1}} & =\sqrt{\frac{\tan \left(\psi_{1}\right)}{\tan \left(\psi_{2}\right)} \frac{G_{L 1}}{G_{L 2}} \frac{\widehat{G}_{m c 1}}{\widehat{G}_{m c 2}}} \\
& \approx 1+\frac{Q\left(\epsilon_{l}+\epsilon_{c}\right)}{2 \zeta}+\frac{1}{2} \eta+\lambda \varepsilon \Leftrightarrow \varepsilon \\
& \approx \frac{Q\left(\epsilon_{l}+\epsilon_{c}\right)}{2(1-\lambda) \zeta}+\frac{\eta}{2(1-\lambda)} \\
\sin (\Delta \hat{\phi}) & =\frac{\frac{\hat{A}_{1}}{\hat{A}_{2}} \frac{\alpha_{2}}{\alpha_{1}}-\frac{\hat{A}_{2}}{\hat{A}_{1}} \frac{\alpha_{1}}{\alpha_{2}}}{\frac{\zeta_{1}}{\mu_{o 2}}\left(\frac{\hat{A}_{1}}{\hat{A}_{2}}\right)^{2} \frac{\alpha_{2}}{\alpha_{1}}+\frac{\zeta_{2}}{\mu_{o 1}}\left(\frac{\hat{A}_{2}}{\hat{A}_{1}}\right)^{2} \frac{\alpha_{1}}{\alpha_{2}}} \\
& \approx-\frac{\mu_{o}}{1-\lambda} \frac{\left(\epsilon_{l}+\epsilon_{c}\right) Q}{2 \zeta^{2}}-\frac{\mu_{o}}{1-\lambda} \frac{\eta}{2 \zeta}-\mu_{o} \frac{\nu}{\zeta} .
\end{aligned}
$$

\section{APPENDIX III}

Fourier transforming the system of (18) and (19) in Section V, one obtains

$$
\begin{aligned}
j x \frac{\widetilde{\delta A_{i}}}{\hat{A}}= & \mu_{o}\left[1-3\left(\frac{\hat{A}}{\alpha}\right)^{2}\right] \widetilde{\delta A_{i}} \\
& -\frac{\widehat{G}_{m c}}{G_{L}}\left(\widetilde{\delta \phi_{j}}-\widetilde{\delta \phi_{i}}\right)-\widetilde{G_{n i}} \\
j x \widetilde{\delta \phi_{i}}= & \frac{\widehat{G}_{m c}}{G_{L}}\left(1-2 \mu_{c}\right) \frac{\widetilde{\delta A_{j}}}{\hat{A}}-\frac{\widehat{G}_{m c}}{G_{L}} \frac{\widetilde{\delta A_{i}}}{\hat{A}}-\widetilde{B_{n i}} \\
& (i, j) \in\{1,2\} ; \quad i \neq j .
\end{aligned}
$$

$$
\begin{aligned}
& \left(G_{L 1}-G_{m o, 1}\right) A_{1}+G_{m c, 2} A_{2} \sin \left(\phi_{2}-\phi_{1}\right)+G_{L 1} \frac{d A_{1}}{d \tau_{1}}=0 \Leftrightarrow \frac{d A_{1}}{d \tau_{1}}=\mu_{o, 1}\left[1-\left(\frac{A_{1}}{\alpha_{1}}\right)^{2}\right] A_{1}-\frac{G_{m c, 2}}{G_{L 1}} \sin \left(\phi_{2}-\phi_{1}\right) A_{2} \\
& -G_{L 1} \tan \left(\psi_{1}\right) A_{1}+G_{m c, 2} A_{2} \cos \left(\phi_{2}-\phi_{1}\right)-G_{L 1} \frac{d \phi_{1}}{d \tau_{1}} A_{1}=0 \Leftrightarrow \frac{d \phi_{1}}{d \tau_{1}}=\frac{2 Q_{1}}{\omega_{01}} \Delta \omega_{1}+\frac{G_{m c, 2}}{G_{L 1}} \cos \left(\phi_{2}-\phi_{1}\right) \frac{A_{2}}{A_{1}}
\end{aligned}
$$




$$
\begin{aligned}
\left\langle\widetilde{\delta A^{2}}\right\rangle= & \frac{\frac{N_{0}}{P_{0}}}{4\left(\mu_{o} x\right)^{2}+16\left(\frac{\widehat{G}_{m c}}{G_{L}}\right)^{4}\left(1-\mu_{c}\right)^{2}}\left\{\frac{4 x^{2} \mu_{o}^{2}+8\left(\frac{\widehat{G}_{m c}}{G_{L}}\right)^{4}}{4 \mu_{o}^{2}}+2\left(\frac{\widehat{G}_{m c}}{G_{L}}\right)^{2}\right\} \\
\left\langle\widetilde{\delta \phi^{2}}\right\rangle= & \frac{\frac{N_{0}}{P_{0}}}{4\left(\mu_{o} x\right)^{2}+16\left(\frac{\widehat{G}_{m c}}{G_{L}}\right)^{4}\left(1-\mu_{c}\right)^{2}} \\
& \times\left\{\frac{8\left(x \mu_{o}\right)^{2}\left(\frac{\widehat{G}_{m c}}{G_{L}}\right)^{2}\left(1+\left(2 \mu_{c}-1\right)^{2}\right)+32\left(\frac{\widehat{G}_{m c}}{G_{L}}\right)^{6} \mu_{c}^{2}\left(1-\mu_{c}\right)^{2}}{4\left(\mu_{o} x\right)^{2}}+\frac{4\left(\mu_{o} x\right)^{2}+8\left(\frac{\widehat{G}_{m c}}{G_{L}}\right)^{4}\left(1-\mu_{c}\right)^{2}}{x^{2}}\right\}
\end{aligned}
$$

The tilde $\sim$ denotes a Fourier transformed variable and

$$
x=\frac{\omega_{m}}{\omega_{3 \mathrm{~dB}}}
$$

where $\omega_{m}$ is the frequency offset from the carrier. The singlesided power/cross-power spectral densities of the normalized noise conductances and susceptances are evaluated to be

$$
\begin{aligned}
\left\langle\widetilde{G_{n, i}}{\widetilde{G_{n, j}}}^{*}\right\rangle & =\left\langle\widetilde{B_{n, i}} \widetilde{B_{n, j}} *\right. \\
& = \begin{cases}\frac{8 k T}{\widehat{A}^{2} G_{L}}=\frac{N_{0}}{P_{0}}, & i=j \\
0, & i \neq j\end{cases} \\
\left\langle\widetilde{G_{n, i}}{\widetilde{B_{n, i}}}^{*}\right\rangle & =\left\langle\widetilde{G_{n, i}} \widetilde{B}_{n, i}\right\rangle=0, \quad i \in\{1,2\}
\end{aligned}
$$

where $\langle\cdot\rangle$ denotes ensemble average, $k$ is Boltzman's constant, and $T$ is the absolute temperature. $N_{0}$ is the noise power, assuming a $1-\mathrm{Hz}$ bandwidth, and $P_{0}$ is the signal power delivered to the load

$$
\begin{aligned}
& N_{0}=4 k T \\
& P_{0}=\frac{\hat{A}^{2} G_{L}}{2} .
\end{aligned}
$$

Equations (42) and (43) can be written in matrix form as

$$
\begin{aligned}
{\left[\begin{array}{c}
\widetilde{G_{n 1}} \\
\widetilde{G_{n 2}} \\
\widetilde{B_{n 1}} \\
B_{n 2}
\end{array}\right]=} & {\left[\begin{array}{cccc}
-2 \mu_{o}-j x & 0 & \frac{\widehat{G}_{m c}}{G_{L}} & -\frac{\widehat{G}_{m c}}{G_{L}} \\
0 & -2 \mu_{o}-j x & -\frac{\widehat{G}_{m c}}{G_{L}} & \frac{\widehat{G}_{m c}}{G_{L}} \\
-\frac{\widehat{G}_{m c}}{G_{L}} & \frac{\widehat{G}_{m c}}{G_{L}}\left(1-2 \mu_{c}\right) & -j x & 0 \\
\frac{\widehat{G}_{m c}}{G_{L}}\left(1-2 \mu_{c}\right) & -\widehat{G}_{m c} & 0 & -j x
\end{array}\right] } \\
\text { where } & \times\left[\begin{array}{c}
\frac{\widehat{\delta A_{1}}}{G_{L}} \\
\frac{\delta A_{2}}{\hat{A}} \\
\widehat{\delta \phi_{1}} \\
\widetilde{\delta \phi_{2}}
\end{array}\right]
\end{aligned}
$$

$$
\mu_{o}\left[1-3\left(\frac{\hat{A}}{\alpha}\right)^{2}\right]=-2 \mu_{o}
$$

is valid for a symmetric circuit. Inverting the $4 \times 4$ matrix in (49) yields

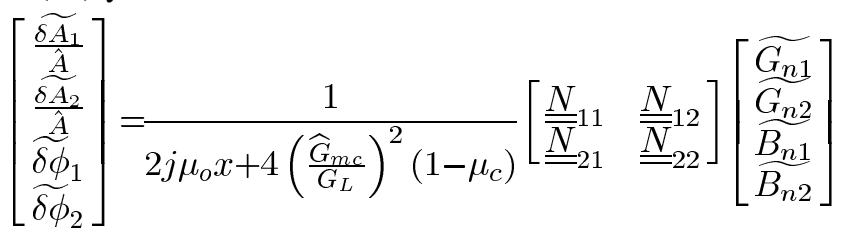

$$
\underline{\underline{N}}_{11}=\left[\begin{array}{cc}
-\frac{2 j x \mu_{o}+2\left(\frac{\widehat{G}_{m c}}{G_{L}}\right)^{2}\left(1-\mu_{c}\right)}{2 \mu_{o}} & -\frac{2\left(\frac{\widehat{G}_{m c}}{G_{L}}\right)^{2}\left(1-\mu_{c}\right)}{2 \mu_{o}} \\
\left.-\frac{2\left(\widehat{G}_{m c}\right.}{G_{L}}\right)^{2}\left(1-\mu_{c}\right) & -\frac{2 j x \mu_{o}+2\left(\frac{\widehat{G}_{m c}}{G_{L}}\right)^{2}\left(1-\mu_{c}\right)}{2 \mu_{o}}
\end{array}\right]
$$$$
\underline{N}_{12}=\left[\begin{array}{cc}
-\frac{\widehat{G}_{m c}}{G_{L}} & \frac{\widehat{G}_{m c}}{G_{L}} \\
\frac{\widehat{G}_{m c}}{G_{L}} & -\frac{\widehat{G}_{m c}}{G_{L}}
\end{array}\right]
$$

$$
\underline{N}_{21}=\frac{\widehat{G}_{m c}}{G_{L}}\left[\begin{array}{ll}
a_{1} & a_{2} \\
a_{2} & a_{1}
\end{array}\right]
$$

$$
\begin{aligned}
a_{1} & =\frac{\left(2 j x \mu_{o}+4 \mu_{c}\left(\frac{\widehat{G}_{m c}}{G_{L}}\right)^{2}\left(1-\mu_{c}\right)\right)}{2 j x \mu_{o}} \\
a_{2} & =\frac{\left(2 j x \mu_{o}\left(2 \mu_{c}-1\right)+4 \mu_{c}\left(\frac{\widehat{G}_{m c}}{G_{L}}\right)^{2}\left(1-\mu_{c}\right)\right)}{2 j x \mu_{o}} \\
\underline{N}_{22} & =\left[\begin{array}{cc}
-\frac{2 j x \mu_{o}+2\left(\frac{\widehat{G}_{m c}}{G_{L}}\right)^{2}\left(1-\mu_{c}\right)}{j x} & -\frac{2\left(\frac{\widehat{G}_{m c}}{G_{L}}\right)^{2}\left(1-\mu_{c}\right)}{j x} \\
-\frac{2\left(\frac{\widehat{G}_{m c}}{G_{L}}\right)^{2}\left(1-\mu_{c}\right)}{j x} & -\frac{2 j x \mu_{o}+2\left(\frac{\widehat{G}_{m c}}{G_{L}}\right)^{2}\left(1-\mu_{c}\right)}{j x}
\end{array}\right] .
\end{aligned}
$$

Equations (51) to (57) specify four transfer functions for each of the stochastic variables $\delta A_{1}, \delta A_{2}, \delta \phi_{1}$, and $\delta \phi_{2} . N_{11}$, $\underline{\underline{N}}_{12}, \underline{\underline{N}}_{21}$, and $\underline{\underline{N}}_{22}$ contain the AM-to-AM, PM-to-AM, $\overline{\mathrm{AM}}$-to-PM, and $\overline{\overline{\mathrm{PM}}} 22$-to-PM transfer functions, respectively. Circuit symmetry is assumed, and we can discard the subscripts as the response will be identical at each node. The input variables are random, so only the magnitude of the transfer functions need be considered. From (46), it is seen that all four random variables are uncorrelated. The amplitude and phase power spectra, assuming a $1-\mathrm{Hz}$ bandwidth, are then derived by summing the contribution from each of the four noise admittances. Using (45), one obtains (58) and (59), shown at the top of the page.

\section{REFERENCES}

[1] A. Rofougaran, J. Rael, M. Rofougaran, and A. Abidi, "A $900 \mathrm{MHz}$ CMOS LC-oscillator with quadrature outputs," in ISSC Tech. Dig. Papers, Int. Conf. Solid State Circuits, 1996, pp. 392-393.

[2] P. Vancorenland and M. Steyaert, "A $1.57-\mathrm{GHz}$ fully integrated very low-phase-noise quadrature VCO," IEEE J. Solid-State Circuits, vol. 37, no. 5, pp. 653-656, May 2002. 
[3] D. Cordeau, J.-M. Paillot, H. Cam, G. De Astis, and L. Dascalescu, “A fully monolithic SiGe quadrature voltage controlled oscillator design for GSM/DCS-PCS applications," in Proc. IEEE RFIC Symp., 2002, pp. $455-458$.

[4] J. van der Tang, P. van de Ven, D. Kasperkovitz, and A. van Roermund, "Analysis and design of an optimally coupled 5-GHz quadrature $L C$ oscillator," IEEE J. Solid-State Circuits, vol. 37, no. 5, pp. 657-661, May 2002.

[5] R. Aparicio and A. Hajimiri, "A noise-shifting differential Colpitts VCO," IEEE J. Solid State Circuits, vol. 37, no. 12, pp. 1728-1736, Dec. 2002.

[6] L. Romano, S. Levantino, A. Bonfanti, C. Samori, and A. L. Lacaita, "Phase noise and accuracy in quadrature oscillators," in Proc. Int. Symp. Circuits Systems, vol. 1, 2004, pp. 161-164.

[7] P. Vanassche, G. Gielen, and W. Sansen, "Behavioral modeling of (coupled) harmonic oscillators," IEEE Trans. Comput.-Aided Des. Integr. Circuits Syss., vol. 22, no. 8, pp. 1017-1026, Aug. 2003.

[8] P. Andreani, A. Bonfanti, L. Romano, and C. Samori, "Analysis and design of a 1.8-GHz CMOS LC quadrature VCO," IEEE J. Solid-State Circuits, vol. 37, no. 12, pp. 1737-1747, Dec. 2002.

[9] J. Wang, J. Tan, and O. Wing, "Theory of cross-coupled RF oscillator for multi- and quadrature-phase signal generation," in Proc. 5th Int. Conf. ASIC, 2003, pp. 1014-1017.

[10] H.-C. Chang, X. Cao, U. Mishra, and R. A. York, "Phase noise in coupled oscillators: Theory and experiment," IEEE Trans. Microw. Theory Tech., vol. 45, no. 5, pp. 604-615, May 1997.

[11] W. C. Lindsey, Synchronization Systems in Communication and Control. Englewood Cliffs, NJ: Prentice-Hall, 1973.

[12] W. A. Edson, "Noise in oscillators," Proc. IRE, vol. 48, pp. 1454-1466, Aug. 1960.

[13] K. Kurokawa, "Noise in synchronized oscillators," IEEE Trans. Microw. Theory Tech., vol. MTT-16, no. 4, pp. 234-240, Apr. 1968.

[14] C. Samori, A. Lacaita, F. Villa, and F. Zappa, "Spectrum folding and phase noise in LC tuned oscillators," IEEE Trans. Circuits Syst. II, Analog Digit. Signal Process., vol. 45, no. 7, pp. 781-790, Jul. 1998.

[15] K. Kurokawa, "Injection locking of microwave solid-state oscillator," Proc. IEEE, vol. 61, no. 10, pp. 1386-1410, Oct. 1973.

[16] G. V. Klimovitch, "Optimization of tunable oscillators with AM to FM conversion for near carrier phase noise," in Proc. RAWCON, 2000, pp. $207-210$.

[17] A. Takaoka and K. Ura, "Noise analysis of nonlinear feedback oscillator with AM-PM conversion coefficient," IEEE Trans. Microw. Theory Tech., vol. MTT-28, no. 6, pp. 654-662, Jun. 1980.

[18] D. Leeson, "A simple model of feedback oscillator noise," Proc. IEEE, vol. 54, no. 2, pp. 429-430, Feb. 1966.

[19] A. Hajimiri and T. H. Lee, "A general theory of phase noise in electrical oscillators," IEEE J. Solid-State Circuits, vol. 33, no. 2, pp. 179-194, Feb. 1998.

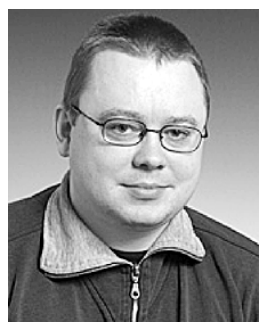

Torsten Djurhuus received the M.S. degree in electrical engineering from the Technical University of Denmark, Lyngby, Denmark, in 2003, where he is currently working toward the Ph.D. degree.

His research interests include nonlinear circuit analysis, MMIC design, and RF oscillator design.

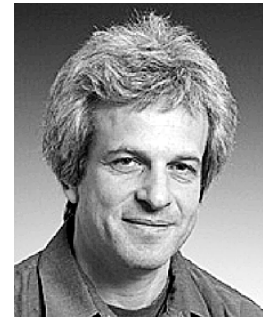

Viktor Krozer (M'93-SM'03) was born in Leningrad, Russia, in 1958. He received the Dipl.-Ing. degree in electrical engineering and the Dr.-Ing. degree (summa cum laude) from the Technische Hochschule Darmstadt (TH Darmstadt), Darmstadt, Germany, in 1984 and 1991, respectively, both in electrical engineering. His dissertation focused on the development of analysis methods for large-signal nonlinear microwave circuits and semiconductor device simulation.

In 1991, he joined the Microwave Electronics Laboratory, TH Darmstadt, as a Senior Research Scientist, where he was involved in the areas of high-temperature semiconductor device operation and submillimeter-wave device development. From 1996 to 2002, he was a Professor of electrical engineering with the Technical University of Chemnitz (TU Chemnitz), Germany, in the area of microwave electronics, where he was the head of the Microwave Laboratory. Since 2002, he has been with the Ørsted-EMI Department, Technical Unviersity of Denmark, Lyngby. His research areas include physical modeling of semiconductor devices and circuits, MMIC and MCM technology, reliability of semiconductor devices, and submillimeter-wave devices and systems. He has contributed to several book chapters and has published more than 100 contributions in international journals and conferences.

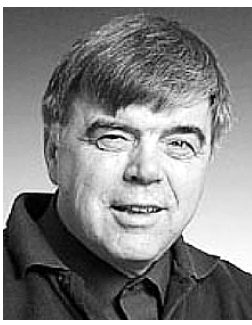

Jens Vidkjær (S'72-M'72) received the M.S. and $\mathrm{Ph} . \mathrm{D}$. degrees from the Technical University of Denmark, Lynbgy, in 1968 and 1975, respectively.

Since 1970, he has been with the Electronics Laboratory and the Semiconductor Laboratory, Technical University of Denmark, where he is presently a Reader with the Electromagnetic System Group of the Ørsted Institute. His research areas have covered RF-power amplifier design, CAD methods, device modeling, measurement accuracies, and MMIC design.

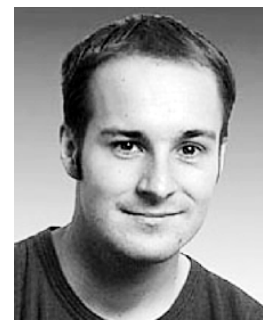

Tom K. Johansen received the M.S. and Ph.D. degrees in electrical engineering from the Technical University of Denmark, Lyngby, in 1999 and 2003, respectively.

In 1999, he joined Ørsted-DTU, Section of Electromagnetic Systems, Technical University of Denmark, where he is currently an Assistant Professor From September 2001 to March 2002, he was a Visiting Scholar with the Center for Wireless Communication, University of San Diego, San Diego, CA. His research areas include the modeling of HBT devices, nonlinear circuit analysis, MMIC design, and electromagnetic simulation. 